\title{
Organisation, fonctionnement et compétence du Tribunal de commerce
}

\author{
Par Elie Katenga Maku*
}

\section{Résumé}

La présente étude est consacrée à l'« organisation, fonctionnement et compétence du Tribunal de commerce ». Son contenu s'articule autour de l'analyse de la législation qui régit le Tribunal de commerce en République Démocratique du Congo. De cette réflexion, il s'est avérée que la loi du 03 juillet 2001 prévoit un régime juridique applicable à la juridiction précitée. La spécialité des règles qui régissent le Tribunal de commerce distingue cette instance juridictionnelle des Tribunaux de Paix et des Tribunaux de Grande Instance dans plusieurs matières, entre autres, la présence des juges consulaires dans la composition des chambres alors qu'ils ne sont pas magistrats. Vingt ans après son application en République Démocratique du Congo, la réalité est que le texte législatif en examen est imprécis et obsolète. L'imprécision réside dans le fait que le législateur prévoit des règles organiques, procédurales et de compétences qui sont identiques tant dans un contentieux pénal que dans un litige civil. L'obsoléité est manifestement palpable d'abord dans le silence du législateur sur plusieurs questions de droit que la législation supranationale renvoie au droit national, en suite dans le contenu de certains articles. Fort de ce constat, il est crucial de procéder à une réforme profonde de la loi du 03 juillet 2001 portant création, organisation et fonctionnement des Tribunaux de commerce afin de rendre précises ses règles organiques, procédurales et de compétences selon que nous sommes en matière pénale ou en matière civile. En outre, la réforme visera aussi à adapter le contenu de la loi précitée au cadre juridique actuel fortement influencé par le droit communautaire.

\begin{abstract}
The current study is entitled 'Organization, functioning and jurisdictions of the Tribunal of Commerce.' The content of this study is focused on the analysis of the legislation which regulates the Tribunal of Commerce. What we can draw from the analysis on the Law of 3 July 2001, which regulates the above-mentioned Tribunal, is that it provides for a
\end{abstract}

* Elie KATENGA MAKU est un Avocat au Barreau de Kinshasa-Matete et Assistant à l'Université Technologique Belcampus. Auxiliaire de Justice depuis le 31/03/2017 où il a commencé sa carrière comme défenseur judiciaire près le Tribunal de Grande-Instance de Kinshasa-Matete, il intégrera une année après le corps des Avocats près la Cour d'Appel de Matete. Auteur d'un article publié en 2019 dans la revue de la Faculté de Droit de l'Université Libre de Kinshasa, Il est en ce moment étudiant en $3^{\text {ème }}$ cycle à l'Université de Kinshasa et enseigne le Droit à l'Université Chrétienne Cardinal MALULA. 
special legal regime to the above-mentioned Tribunal. The specialty of rules regulating the Tribunal of Commerce distinguish between the latter Tribunal and the other Tribunals like the Tribunal de paix and High Courts in many matters such as for instance the presence of consular judges sitting on the bench while they are not magistrates. Twenty years later after its application in the Democratic Republic of the Congo, we can say that the legislative text in question is in accurate and obsolete. The inaccuracy resides in the fact that the law-maker provides for organizational, procedural rules and court jurisdictions which are identical in both criminal matter and civil matters. The obsolete element is clearly existent in the silence of the law-maker on many questions of law which the supranational legislation sends back to the national law, and secondly on the content of certain articles. With this strong assessment, it is very relevant to proceed with an important reform of the Law of 3 July 2001 establishing the organizational and functioning of tribunals of commerce in order to make the organizational and procedural rules and court jurisdictions more accurate when we deal with criminal and civil matters. Further, the reform will aim at adapting the concerned law to the current legal framework which is strongly influenced by the Communal Law.

\section{Introduction}

L'exposé des motifs de la Constitution du 18/02/2006 renseigne que les Cours et Tribunaux en RDC sont éclatés en trois ordres, à savoir : les juridictions de l'ordre judiciaire, les juridictions de l'ordre administratif et la Cour constitutionnelle ${ }^{1}$. Au sein de l'ordre judiciaire congolais, le législateur a institué depuis $2001^{2}$, une instance juridictionnelle chargée de régler les litiges commerciaux, laquelle instance porte le nom de « Tribunal de commerce ».

La présente étude s'articule autour du régime juridique spécial institué par le pouvoir législatif en rapport avec l'organisation, le fonctionnement et la compétence du Tribunal de commerce. La juridiction précitée est régie par des règles juridiques particulières qui la différencient du Tribunal de Grande Instance et du Tribunal de Paix. Il convient d'identifier quelques-unes de ces règles et de ressortir les différentes failles constatées dans ce système dérogatoire en vigueur depuis 2001.

\section{A. ANALYSE DU REGIME JURIDIQUE APPLICABLE AUX TRIBUNAUX DE COMMERCE}

L'article 2 de la loi n002-2001 du 03/07/2001 portant création, organisation et fonctionnement des Tribunaux de commerce dispose que : le tribunal de commerce est une juridiction

1 Exposé des motifs de la Constitution de la République Démocratique du Congo du 18/02/2006 telle que révisée par la loi n¹1/002 du 20/01/2011, Kinshasa, JORDC, Numéro spécial 05/02/2011.

2 « Il est crée des tribunaux de commerce en République démocratique du Congo » Art. 1 de la Loi $\mathrm{n}^{\circ} 002-2001 \mathrm{du}$ 03/07/2001 portant création, organisation et fonctionnement des Tribunaux de commerce, Kinshasa, JORDC, n¹4, 15/07/2001, p.4. 
de droit commun siégeant au premier degré et composée de juges permanents qui sont des magistrats de carrières et des juges consulaires. Son siège ordinaire et son ressort sont ceux du tribunal de grande instance. Il est présidé par un magistrat du siège appartenant au corps judiciaire désigné et, le cas échéant, relevé de ses fonctions le ministre de la justice ${ }^{3}$.

La doctrine congolaise ne donne pas la connotation du Tribunal de commerce. MATADI NENGA renseigne, néanmoins, que la juridiction susvisée a un caractère spécial ${ }^{4}$. Pour la doctrine Française, le TRICOM est une juridiction spécialisée du premier degré de l'ordre judiciaire établie dans les circonscriptions où une suffisante activité commerciale le justifie, composée des membres élus par un collège électoral constitué des représentants de professions commerciales et principalement compétente pour juger les contestations relatives aux engagements entre commerçants, des contestations entre associés, de celles aux actes de commerce 5 .

La définition de Gérard CORNU est propre au Droit Français. En RDC, le Tribunal de commerce n'est pas composé que des professionnels. Les magistrats de carrière aussi participent à la distribution de la justice que réalise cette juridiction. Le point commun entre les deux législations est la reconnaissance de la nature particulière du TRICOM. Le caractère spécial du Tribunal de commerce, rappelé par la doctrine congolaise, se situe aux niveaux organisationnel, fonctionnel et à la délimitation de sa compétence matérielle.

Ainsi, le point A de la présente étude est subdivisée en trois sous-points consacrés, respectivement, à la:

- Spécialité du Régime organisationnel du Tribunal de commerce;

- Spécialité du Régime fonctionnel du Tribunal de commerce;

- Spécialité des règles de compétence du Tribunal de Commerce.

\section{SPECIALITE DANS LE REGIME ORGANISATIONNEL}

Un Tribunal en RDC est composé des magistrats de carrière, des greffiers et des fonctionnaires de l'Etat affectés par les pouvoirs publics pour exercer une mission d'intérêt général. La direction d'une juridiction de cette nature est confiée à un magistrat de carrière appelé Président, lequel bénéficie d'un dédoublement fonctionnel puisqu'il est à la fois autorité administrative et judiciaire. La première fonction se limite aux mesures d'ordre intérieur au sein de la juridiction. La deuxième va au-delà des simples mesures d'hygiène et de sécurité au lieu de travail. Elle touche l'aspect juridictionnel, mieux, la mission de dire le droit.

La spécialité du régime organisationnel du Tribunal de commerce se décèle par la présence des personnes étrangères à la magistrature, mais qui siègent à côté des magistrats de carrière dans les litiges commerciaux. Il s'agit des juges consulaires. Ces derniers sont

3 Art. 2 de la loi n002-2001 du 03/07/2001 portant création, organisation et fonctionnement des Tribunaux de commerce, Kinshasa, JORDC, n¹4, 15 Juillet 2001, p.4.

4 MATADI NENGA GAMANDA, «Droit judiciaire privé », Kinshasa, 2006, pp.78 - 79.

5 Gérard CORNU, « Vocabulaire juridique », Paris, 2014, p.1040. 
élus par les délégués consulaires des organisations professionnelles des commerçants et des industriels d'une province donnée. Après le vote, le Ministre de la justice entérine le contenu du procès-verbal du scrutin.

L'élection des juges consulaires est coordonnée par le bureau du collège électoral dans lequel se réunissent les émissaires des structures locales spécialisées dans le business. Dirigé par le doyen des représentants de ces organisations, le bureau examine le dossier des candidats juges consulaires afin de vérifier s'ils sont éligibles au regard de l'article 9 de la loi du 03/07/2001 portant création, organisation et fonctionnement des Tribunaux de commerce $^{6}$. Après son élection et entérinement du Ministre de la Justice, les susdits juges consulaires prêtent serment devant le Tribunal de Commerce, à défaut, devant le Tribunal de Grande Instance du ressort.

Le législateur exclut le juge consulaire de la direction du Tribunal de commerce. Seul le magistrat de carrière est autorisé à diriger le TRICOM, seul lui préside les chambres, seul lui engage sa responsabilité professionnelle devant le Conseil supérieur de la magistrature et devant la Cour de cassation en procédure de prise à partie ${ }^{7}$. Le juge consulaire qui, a un mandat de deux ans pour sa première élection et quatre pour les autres avec restriction de ne pas faire plus de trois mandats dans une même juridiction, compose la formation collégiale du Tribunal dans les audiences.

Il est totalement exclu dans le contentieux où le législateur désigne expressément le magistrat comme seul organe habilité à statuer. Tel est le cas des contestations des saisies conservatoires et exécutions prévues par l'Acte uniforme du 10/04/1998 portant organisation des procédures simplifiées de recouvrement et de voies d'exécution. L'article 49 al. $1^{\text {er }}$ de l'instrument communautaire précité confie la fonction de juge d'exécution au Président des juridictions qui ne peut être qu'un magistrat de carrière. En cas d'empêchement, le chef d'office ne délègue ce pouvoir qu'à un magistrat, une façon pour le législateur supranational d'écarter les non-magistrats de ce contentieux ${ }^{8}$.

6 «Sont éligibles aux fonctions de juge consulaire, les Congolais âgé de trente ans au moins et de soixante ans au plus, ayant pendant cinq ans au moins, honorablement exercé le commerce ou participé soit à la gestion d'une société commerciale de droit congolais, en ce compris les associés des sociétés en nom collectif et en commandite simple et les administrateurs actifs ou gérants des sociétés à responsabilité limitée, soit à la direction d'une organisation professionnelle ou interprofessionnelle représentative du commerce et de l'industrie ainsi que les cadres supérieurs et conseils juridiques des sociétés et chambre de commerce. Sont inéligibles, les candidats frappés par les cas de déchéance ou d'incompatibilité visés à l'article 4 de l'ordonnance 79-025 du 07 février 1979 relative à l'ouverture d'un nouveau registre de commerce » Art. 9 de la Loi n ${ }^{\circ} 002-2001$ portant création, organisation et fonctionnement des Tribunaux de commerce, Kinshasa, JORDC, Numéro spécial, 15/07/2001, p.4.

7 «Tout magistrat de l'ordre judiciaire peut être pris à partie dans les cas suivants... » Art. 55 de la Loi-organique $\mathrm{n}^{\circ} 13 / 010 \mathrm{du}$ 19/02/2013 relative à la procédure devant la Cour de cassation, Kinshasa, JORDC, Numéro spécial, 20/02/2013.

8 « La juridiction compétente pour statuer sur tout litige ou toute demande relative à une mesure conservatoire est le président de la juridiction statuant en matière d'urgence ou le magistrat délégué par lui » Art. 49 de l’Acte uniforme du 10/04/1998 portant Organisation des Procédures Simpli- 
A ce jour, la présence des experts du monde des affaires dans la composition des chambres constitue une spécialité remarquable dans le régime organisationnel des Tribunaux de commerce. La recherche de la technicité dans le règlement des litiges commerciaux a motivé le législateur à faire appel aux spécialistes du business law pour venir épauler les magistrats dans la gestion des actions juridictionnelles.

Le parquet, lui, n'a pas été touché par la loi du 03/07/2001 portant création, organisation et fonctionnement des Tribunaux de commerce. Le Procureur près le Tribunal de Grande Instance exerce la mission d'officier du ministère public devant le Tribunal de commerce. Certains juristes jugent nécessaire la création d'un parquet spécialisé en matière commerciale. L'argument évoqué s'articule autour de la formation universitaire des licenciés congolais, laquelle formation est générale. Comme qui dirait, lorsqu'on est roi de tout, on est roi sans royaume. Les magistrats debout se heurtent à des difficultés réelles dans les analyses des dossiers qui leur sont soumis, surtout en matière civile où ils doivent émettre un avis sur le banc en vue de favoriser la célérité dans la clôture des causes inscrites sur le rôle à plaider. Ils préfèrent demander le dossier en communication pour coucher leur position sur un papier.

Dans le contentieux non pénal, le Ministère public intervient par voie d'avis, lequel est émis soit sur le banc quelques minutes après la plaidoirie des parties, soit par écrit dans un délai de trente jours après avoir reçu le dossier des mains du greffier du Tribunal. Le deuxième cas est généralement utilisé lorsque le magistrat dépêché scrute la cause le même jour et estime nécessaire de plus réfléchir avant de donner son point de vue. Devant le Tribunal de commerce, cette hypothèse est devenue la règle en raison de la technicité des causes qui sont soumises à cette juridiction.

A notre avis, la création des parquets spéciaux est utile, mais pas nécessaire. D'autres solutions peuvent être imaginées pour pallier à cette difficulté. Il s'agit notamment de l'application de l'article 44 de l'Arrêté d'organisation judiciaire du 20/08/1979 qui dispose : "les parties pourront déposer au greffier leurs conclusions écrites pour qu'elles soient par le greffier communiquées au Ministère public afin de lui permettre de prendre connaissance des litiges dans lesquels il estime son intervention utile ou nécessaire ${ }^{9}$. Cette disposition a le mérite de mettre les justiciables devant leur responsabilité en ce qu'ils peuvent remettre le dossier au greffier afin que ce dernier l'envoie au parquet pour un avis à émettre sur le banc.

En matière civile, les parties ont jusqu'à trois remises avant la plaidoirie. Le premier renvoi est d'un mois, le deuxième est de deux semaines et le troisième est souvent de trois semaines pour une sommation de conclure. Pendant que les conseils s'échangent les pièces et conclusions, ils gagneront en temps en déposant le dossier au greffe pour

fiées de Recouvrement des créances et des Voies d'Exécution, In JO/OHADA, n06,01/06/1998,p.1 et s.

9 Art. 45 de 1'Arrêté d'Organisation Judiciaire n²99/79 du 20/08/1979 portant règlement intérieur des cours, tribunaux et parquets. 
une communication au ministère public avant l'audience de la plaidoirie. Dans ce cas, le parquet examinera suffisamment la cause dans le délai et émettra son avis sur le banc.

L'inopportunité d'un parquet économique est aussi grande en matière pénale qu'en matière civile. Le droit pénal des affaires a la particularité de réduire le pouvoir du Ministère public dans sa mission de rechercher les infractions et leurs auteurs. Le Procureur est généralement assisté des agents des services publics spécialisés qui ont la qualité d'officier de police judiciaire. Tel est le cas des agents de la Banque Centrale du Congo pour les infractions en matière bancaire et cambiaire, les fonctionnaires de la Direction Générale des Impôts pour les incriminations fiscales pour ne citer que ces deux cas là.

L'expertise des services spéciaux dont bénéficie le parquet durant la phase pré juridictionnelle enlève à l'OMP la possibilité d'évoquer la technicité de la matière comme blocage à l'action publique. Un travail technique des OPJ spéciaux est mis à sa disposition avant la saisine de la juridiction répressive. En se basant sur le rapport des agents spécialisés, le parquet n'aura plus qu'à apprécier l'opportunité de saisir ou pas le Tribunal. Fort de cette évidence, il n’y a donc pas urgence de créer un parquet commercial. Ce qui est urgent, c'est l'installation des parquets sur l'ensemble du pays et l'affectation des magistrats compétents dans ces différents offices.

\section{SPECIALITE DANS LE REGIME FONCTIONNEL}

Les règles régissant le fonctionnement d'une juridiction prennent en compte d'une part le statut de service public dont jouit l'instance juridictionnelle en examen, et d'autre part, sa mission particulière qui consiste à régler le litige. En droit congolais, le régime fonctionnel des Tribunaux a comme base textuelle l'Arrêté d'organisation judiciaire n²99/79 du 20 août 1979 portant règlement intérieur des Cours, Tribunaux et parquets et les autres textes législatifs, entre autres, la loi-organique du 11/04/2013 portant organisation, fonctionnement et compétence des juridictions de l'ordre judiciaire et la loi du 03/07/2001 portant création, organisation et fonctionnement des Tribunaux de commerce.

Nous resterons plus dans l'aspect procédural où on retrouve quelques caractères intrinsèques au TRICOM. La procédure devant le Tribunal de commerce commence en matière civile soit par requête écrite ou verbale, soit par assignation, conformément au Code de procédure civile. En matière pénale, la citation à prévenu et la citation directe sont les deux modes de saisine de cette instance juridictionnelle à en croire le législateur de 2001. Il faudra aussi ajouter la comparutiosn volontaire qui est un mode de saisine du Tribunal dans une action civile ou criminelle ${ }^{10}$.

La requête est un acte des parties. Devant les Tribunaux ordinaires, elle est souvent employée pour les actions gracieuses et les procédures préalables au litige, entre autres, la tentative de conciliation en matière de divorce. En l'instaurant comme une pièce pouvant

10 Art. 19 et 20 de la loi n002-2001 du 03/07/2001 portant création, organisation et fonctionnement des Tribunaux de commerce, Kinshasa, JORDC, n¹4, 15 Juillet 2001, p.4. 
saisir le Tribunal de commerce dans un contentieux, le législateur congolais a érigé un régime spécial dans le fonctionnement de cette juridiction. Ainsi, à l'instar des actions sociales, les actions contentieuses en matière commerciale peuvent être initiées par une requête.

Les justiciables ont donc la possibilité de choisir la requête ou l'assignation pour lancer la procédure. Dans la première hypothèse le requérant a l'obligation d'envoyer une convocation qui devra remplir les conditions d'une assignation. Cette particularité est la même au Tribunal du Travail.

En sus, le régime fonctionnel du TRICOM donne un délai de huit jours aux juges pour rendre son verdict. La huitaine fixée par le législateur est difficilement respectée par les Tribunaux de commerce congolais en raison des prescrits de l'article 43 de la loi-organique du 11/04/2013 qui établit d'une part une période maximum de trente jours pour les affaires civiles, commerciales et sociales, et d'autre part, un délai maximum de dix jours pour les causes pénales ${ }^{11}$.

Fort malheureusement, dans la pratique, les Tribunaux de commerce observent trente jours avant de rendre un jugement en matière civile en lieu et place de huit jours fixés par la loi de 2001. Cette attitude est préjudiciable aux justiciables qui doivent longuement patienter avant d'obtenir la décision qui leur permet de continuer leurs activités professionnelles. La lenteur constatée au TGI était le motif principal de la création des TRICOMS, il est donc regrettable de voir la même lenteur continuer dans une instance juridictionnelle créée dans le but de permettre aux commerçants de vider rapidement leur litige.

En troisième et dernier lieu vient le délai d'opposition et d'appel. Il est aussi global, il ne diffère guère selon que nous sommes en matière pénale ou en matière civile. Pour le législateur, le justiciable condamné par défaut a huit jours après la signification du jugement pour faire opposition en lieu et place de 15 dans un litige civil ou de 10 en droit pénal de forme. Le même délai s'applique pour une partie qui veut se pourvoir en appel contre une décision contradictoire. Il est aussi important de souligner que les délais d'appel et d'opposition précités s'appliquent rarement dans les litiges prévus par le droit OHADA pour la simple et bonne raison que le législateur communautaire indique lui-même le délai de recours.

\section{SPECIALITE DANS LES REGLES DE COMPETENCE}

En droit judiciaire congolais, il existe trois facteurs qui déterminent la compétence d'une juridiction. Les susdits facteurs sont : l'espace, la matière et la personne, principalement le défendeur. Pour une action civile, le Tribunal est territorialement compétent en fonction soit du domicile de l'assigné ou de l'un des assignés, soit de la localisation de l'immeuble lorsque la cause porte à titre principal sur un bien immobilier. Pour un contentieux pénal,

11 Art. 43 de la loi-organique $n^{\circ} 013 / 11-B$ du 11/04/2013 portant organisation, fonctionnement et compétence des juridictions de l'ordre judiciaire, Kinshasa, JORDC, Numéro spécial du 04/05/2013. 
c'est le lieu de la commission de l'infraction, de l'arrestation du délinquant ou du domicile de ce dernier.

La compétence matérielle est déterminée, dans un litige pénal, par le nombre maximum de la peine légalement établie par le législateur. A cela s'ajoute, le pouvoir de la Cour d'Appel de juger les crimes qui relèvent de la Cour pénale Internationale. Pour le contentieux civil, le juge est compétent en raison du montant des dommages-intérêts et de l'objet de la cause. Enfin, le droit pénal de forme détermine le pouvoir des juridictions selon la situation personnelle du délinquant.

A l'instar des régimes organisationnel et fonctionnel, les règles de compétence prévues par le législateur en matière commerciale sont caractérisées par une spécialité qui se matérialise dans le contenu de l'article 17 de la loi du 03/07/2001 portant création, organisation et fonctionnement des Tribunaux de commerce. Sur pied de la disposition susmentionnée le Tribunal de commerce connaît en matière de droit privé :

- Les contestations relatives aux engagements et transactions entre commerçants;

- Des contestations entre associés pour raisons de société de commerce;

- Des contestations entre toutes personnes relatives aux actes de commerce en ce compris les actes relatifs aux sociétés commerciales, aux fonds de commerce, à la concurrence commerciale et aux opérations de bourse;

- Des actes mixtes si le défendeur est commerçant, des litiges complexes comprenant plusieurs défendeurs dont l'un est soit caution, soit signataire d'un chèque bancaire, d'une lettre de change ou d'un billet;

- Des litiges relatifs aux faillites et concordats judiciaires ${ }^{12}$.

L'énumération faite par le législateur est à la fois objective et subjective. La loi détermine les causes où la compétence du Tribunal de commerce est indubitable nonobstant le statut des justiciables. Il s'agit du deuxième, troisième et dernier point de l'article 17. A l'exception de la société en nom collectif et la société en commandite simple uniquement pour l'associé commandité, la qualité de commerçant n'est pas une condition pour devenir actionnaire ou associé dans une entreprise. Les associés non commerçants soumettront leur contentieux au TRICOM en raison non pas de leur statut mais du caractère commercial des actes qu'ils posent au sein de la société.

En vertu du principe de la commercialité par la forme, une personne morale est commerçante dès lors qu'elle adopte une de cinq formes de sociétés commerciales prévues par l'Acte uniforme du 30/01/2014 relatif aux Sociétés commerciales et Groupement d'Intérêt économique. Ainsi, toutes les opérations passées par les associés sont soumises au droit commercial dès lors qu'elles se rapportent à l'entreprise, de ce fait, le Tribunal de commerce est compétent pour trancher le litige issu des susdites opérations au regard de leur caractère économique.

12 Art. 17 de la Loi $n^{\circ} 002-2001$ portant création, organisation et fonctionnement des Tribunaux de commerce, JORDC, Numéro spécial, 15/07/2001, p.4. 
Le pouvoir du juge est aussi objectif dans la cause ayant trait aux actes de commerce et ce, sans distinction de la commercialité ou pas du justiciable. A l'instar des faillites et concordats judiciaires qui bénéficient même d'un greffe au sein du Tribunal de commerce de Kinshasa-Gombe, les actions nées des actes des sociétés commerciales, des bourses et autres matière prévues aux points trois sont jugées par le Tribunal de commerce pour la simple et bonne raison qu'il s'agit pour la plupart des actes de commerce par nature, lesquels font appel au droit commercial peu importe la qualité de l'auteur.

La compétence subjective du Tribunal de commerce est assise sur les points un et quatre de l'article 17 de la loi du 03/072001 en ce que le législateur fait du statut commercial du défendeur ou de l'un de défendeur un facteur qui permet de retenir la ratio materiae de la juridiction précitée. De la même manière que le pouvoir objectif du TRICOM se rapproche des actes de commerce par nature, lesquels sont régis par le droit commercial peu importe la qualité de leurs auteurs, de la même manière, les attributions dites subjectives se rattachent aux actes de commerce par relation. Il s'agit en réalité des opérations qui, au départ, sont civiles mais deviennent commerciales pour la simple et bonne raison qu'elles sont accomplies par les commerçants au profit de leur activité économique.

La loi ne dit mot sur la compétence territoriale des Tribunaux de commerce. Les règles y afférentes ne sont nullement teintées d'une quelconque spécialité. Elles suivent les principes posés par les dispositions de la loi-organique du 11/04/2013 portant organisation, fonctionnement et compétence des juridictions de l'ordre judiciaire. C'est là la manifestation de la règle selon laquelle le spécial est compris dans le général, mieux, lorsque la législation particulière ne dit mot, la législation générale s'applique de facto. Nous ne pouvons clore ce propos sans évoquer les attributions du Tribunal de commerce en matière pénale.

Le dernier alinéa de l'article 17 de la loi du 03 juillet 2001portant création, organisation et fonctionnement stipule que cette juridiction connaît en matière de droit pénal, des infractions à la législation économique et commerciale, quel que soit le taux de la peine ou la hauteur de l'amende. Contrairement aux juridictions ordinaires où le taux de la peine détermine aussi le juge compétent, le Tribunal de commerce statue sur toutes les actions pénales basées sur la violation du droit des affaires peu importe la sanction répressive y afférente. Il y a nécessité de s'interroger sur le contenu du terme « infractions à la législation économique et commerciale » puisque le législateur n'en donne aucune définition alors que la RDC ne possède pas un code pénal économique.

Doit-on se limiter à affirmer que les termes susvisés renvoient d'une part aux actes criminels posés par les commerçants en raison de la compétence subjective, et d'autre part, aux infractions réalisées dans l'accomplissement des opérations juridiques citées aux points deux, trois et derniers de l'article 17; nenni. Le droit pénal économique va plus loin que ça. Il touche à la fois les incriminations prévues de façon expresse par la législation des affaires et celles établies par les textes répressifs classiques dès lors qu'elles perturbent l'ordre public économique. Le contenu de cette disposition démontre à suffisance que les règles applicables au Tribunal de commerce sont imprécises et obsolètes. 


\section{B. IMPRECISION ET OBSOLEITE DE LA LEGISLATION SUR LE TRIBUNAL DE COMMERCE}

De l'analyse du régime juridique applicable aux Tribunaux de commerce, il sourd que la législation en la matière est devenue imprécise et obsolète après près de vingt ans d'application. Ce constat résume, à notre avis, la majorité pour ne pas dire la totalité des failles décelées dans la loi du 03/07/2001 portant création, organisation et compétence du Tribunal de commerce. Nous aborderons dans les lignes qui suivent les deux résultats de notre analyse.

\section{B IMPRECISION DE LA LEGISLATION SUR LE TRIBUNAL DE COMMERCE}

Une législation élaborée dans le but de régenter l'organisation, le fonctionnement et la compétence d'une juridiction est un instrument juridique de nature à la fois organique et procédural. Dans ce domaine deux théories gouvernent l'interprétation des dispositions en la matière. Il s'agit des adages : " ubi lex distinguit, non distinguere debemus » (là où la loi ne distingue pas, nous ne devons pas distinguer) et « ubi lex voluit, dixit. Ubi lex noluit, tacuit » (là où la loi veut, elle le dit, là où la loi ne veut pas quelque chose, elle se tait) ${ }^{13}$. Comment ne pas distinguer dans un cadre où la loi ne clarifie pas sa position? Comment identifier la volonté ou le refus du législateur dès lors qu'il brille par une superficialité?

Le texte législatif en examen est imprécis sur l'organisation, le fonctionnement pris dans le volet procédural ainsi que sur la compétence du Tribunal de commerce en matière civile et surtout en matière pénale. D'aucuns diraient que les actes réglementaires, la jurisprudence et d'autres sources de droit sont là pour éclairer les zones d'ombre. Sans négliger l'apport des susdites sources, nous estimons qu'il est convenable de laisser le législateur régir à son niveau les faits qui contribuent à la perturbation du quotidien de la vie des affaires. Les dix dernières années ont démontré à quel point le règlement peut créer une insécurité juridique et judiciaire dans un pays.

La jurisprudence, quant à elle, n'est pas une source formelle du droit congolais. Elle ne jouit pas d'une force contraignante sur les magistrats et les justiciables, de ce fait, sa contribution est faible dans l'édifice d'un Etat de Droit. Car ici, les règles seules, gouvernent la marche de la République. De l'administrant à l'administré, du gouvernant au gouverné, les normes sont prétablies en vue de permettre aux différents sujets de droit de prendre les mesures nécessaires pour se mettre en ordre. Dans un pays qui se réclame de la famille romano-germanique comme le nôtre, les décisions juridictionnelles ne s'imposent pas sauf en cas de cassation avec renvoie. C'est pourquoi, nous restons extrêmement attachés au contenu des lois comme c'est le cas avec la loi du 03/07/2001.

Sur le plan organisationnel, la législation en concerne ne précise pas le statut du juge consulaire. Est-il un consultant appelé à assister le magistrat de carrière dans la prise des

13 Emmanuel-Janvier LUZOLO BAMBI LESSA, " Manuel de Procédure Pénale », Kinshasa, 2011,p.59. 
décisions ou un membre effectif de l'organigramme du Tribunal de commerce? Difficile de donner une réponse à ces interrogations car la loi elle-même est muette. Dans la pratique, les juges consulaires n'interviennent pas dans la direction de la juridiction en examen. Ils sont plus présents dans la formation des chambres collégiales. Seul le magistrat de carrière dirige le TRICOM et il est secondé toujours par un autre plus séant.

La question statutaire n'est pas l'unique problématique née de la présence du juge consulaire au sein du Tribunal de commerce. Il y a aussi un autre problème non résolu par le législateur, celui du contrôle de ce dernier dans sa mission de dire le droit. Si le magistrat de carrière est sanctionné par la Cour de cassation via la prise à partie, le juge consulaire lui n'est pas soumis à cette procédure pour la simple et bonne raison qu'il n'est pas un magistrat. Oui, l'article 2 de la loi-organique du 11/04/2013 portant organisation, fonctionnement et compétence des juridictions de l'ordre judiciaire cite le juge du Tribunal de commerce dans le bloc de personnes pouvant être appelées " magistrat »; mais il s'agit du juge au sens strict du terme, celui de la catégorie huit du tableau qui reprend les grades d'un magistrat de carrière.

Le juge consulaire ne figure pas dans la hiérarchie des grades. Son statut est difficile à cerner à l'instar même de l'autorité habilitée à le sanctionner en cas de concussion, déni de justice ou dol commis dans l'exercice de ses fonctions. Alors que le jugement est le résultat de la délibération faite entre un magistrat de carrière et deux juges consulaires, le législateur a décidé de laisser seul le président de la chambre répondre des failles de l'œuvre commune devant la Cour de cassation dans une procédure de prise à partie. C'est un motif d'insécurité judiciaire de laisser à l'appréciation des susdits juges un litige pénal ou civil dès lors que ces derniers ne répondent pas de leurs actes devant une instance technique comme la Haute Cour.

Leur rédevabilité est dirigée vers les organisations professionnelles spécialisées dans le commerce et l'industrie qui leur donnent des voix pour siéger comme juge consulaire et bénéficier des avantages y afférents. Nous savons que les structures des commerçants sont généralement composées des opérateurs économiques financièrement forts qui sont eux-mêmes justiciables devant l'instance juridictionnelle dans laquelle ils ont placé les juges. Bien que la théorie de l'ingratitude existe, les juges consulaires, irresponsables professionnellement, chercheront à assurer les intérêts des électeurs en lieu et place des justiciables. Cette partialité peut négativement impacter le travail du magistrat de carrière qui dirige la chambre, par ricochet, préjudicier les parties au procès. Il est donc primordial que le législateur réfléchisse profondément sur le statut du juge consulaire, sa responsabilité professionnelle ainsi que le contrôle de son activité.

En sus de la mission du juge consulaire, le législateur doit aussi clarifier la procédure devant le Tribunal de commerce selon qu'il s'agit d'un processus civil ou répressif. La législation sur la juridiction précitée pèche par une globalisation des règles procédurales qui, généralement, obstrue les normes qui gouvernent le droit pénal de forme au profit du processus civil tel que prévu par le Décret du 07/03/1960 portant Code de procédure civile. 
Pour s'en convaincre, il suffit de lire l'article 43 de la loi du 03/07/2001 qui fixe à dix ans la prescription des actions nées du fait de commerce ${ }^{14}$.

Le contentieux issu du commerce n'est pas toujours civil. Devant le Tribunal de Commerce se tiennent les audiences qui statuent sur les causes civiles et les affaires pénales. C'est à ce titre que dans cette juridiction, il existe un greffe chargé d'enrôler les affaires commerciales à caractère répressif appelé greffe pénal économique. Les actions de ce genre ne se prescrivent pas par dix ans. Généralement, la législation économique congolaise est muette sur le délai de prescription des infractions propres au droit des affaires. Fort de ce silence, les Cours et Tribunaux saisis des infractions économiques préfèrent suivre les règles de prescriptions retenues par le droit pénal général en lieu et place de l'article 43.

Le délai de dix ans est plus retenu pour les actions civiles que pénales, chose qui constitue une insécurité pour les justiciables qui, par mégardes se fieront aux dires du législateur de 2001. Le risque serait de voir un commerçant être débouté par une juridiction qui a suivi la prescription retenue par le droit pénal général alors que le requérant s'est fondé sur le délai décennal prévu par la loi du 03/07/2001. Cette hypothèse est fort probable en raison de la nature des sanctions prévues par le législateur congolais contre les infractions économiques.

En effet, dans les affaires, les pouvoirs publics prévoient généralementdes sanctions financières et professionnelles (interdiction de gérer, fermeture) en lieu et place de l'emprisonnement comme c'est le cas avec les crimes de droit commun. La combinaison de cette évidence avec le délai de prescription prévu dans notre législation met en exergue la conclusion selon laquelle la majorité des infractions économiques s'éteindront par prescription rapidement si elles sont soumises aux règles du droit pénal général en ce que le Code pénal congolais établit une période d'un an pour les infractions dont la peine varie entre l'amende et la servitude pénale annuelle, trois ans pour les infractions dont le maximum de la servitude pénale est de cinq ans, et enfin, dix ans pour les crimes dont la sanction va de plus de cinq ans à la peine de mort ${ }^{15}$.

L'imprécision est malheureusement perceptible dans les autres dispositions de la loi de 2001. Plusieurs questions de procédure devant le Tribunal de commerce sont traitées de façon globale sans distinction aucune du processus pénal et de l'action civile. Qu'il s'agisse du contenu d'un jugement rendu par le $\operatorname{TRICOM}^{16}$, du préalable pour faire appel

14 «Sauf prescription plus courte prévue par la loi particulière, les actions ayant pour cause les faits du commerce se prescrivent par dix ans, après le fait qui a donné naissance à l'action » Art. 43 de la Loi n ${ }^{\circ} 002-2001$ portant création, organisation et fonctionnement des Tribunaux de commerce, Kinshasa, $J O / R D C, \mathrm{n}^{\circ} 14,15 / 07 / 2001, \mathrm{p} .4$.

15 NYABIRUNGU mwene SONGA, « Droit pénal général zaïrois », Kinshasa, 1989, pp.343 - 344.

16 L'article 33 de la Loi du 03/07/2001 portant création, organisation et fonctionnement des Tribunaux de commerce énumère les éléments qui composent un jugement rendu par le Tribunal de commerce. Cette énumération correspond au jugement civil et s'écarte de l'article 87 du Décret du 06/08/1959 portant Code de procédure pénale en ce que la loi de 2001 ne cite pas l'indication des faits mis à charge sur les prévenus, exposé sommaire des actes de poursuites et de procédure à l'audience, les conclusions éventuelles qui sont les composants d'un jugement pénal. 
ou opposition ${ }^{17}$, du délai même de ces deux voies de recours selon que le pourvoi est intenté par un justiciable ordinaire ou le magistrat du parquet dont le délai d'appel est différent de celui des autres parties dans un procès répressif; toutes ces thématiques et tant d'autres encore sont ignorées par le législateur qui érige un régime juridique unique proche de la procédure civile et distant du contentieux pénal.

La lecture de la loi de 2001 donne l'impression que le Tribunal de commerce est une juridiction qui ne juge que le dossier civil. Nenni, la juridiction en concerne applique le droit pénal de forme. Malheureusement, les normes qui la régissent brillent par une forme d'ignorance de cette évidence. Il appartient donc au législateur de réformer le contenu des dispositions de la loi du 03 juillet 2001 afin de prévenir l'arbitraire.

\section{OBSOLEITE DE LA LEGISLATION SUR LE TRIBUNAL DE COMMERCE}

La norme juridique créant le Tribunal de commerce a été promulguée en 2001. Vingt ans après, le droit congolais a subi plusieurs mutations qui rendent certaines dispositions de la loi du 03/07/2001 obsolète. La plus grande mutation est l'adhésion de la République Démocratique du Congo au Traité du 17/10/1993 relatif à l'harmonisation du droit des affaires en Afrique. La convention internationale précitée a le mérite d'avoir créé une organisation communautaire dont le but est de mettre en place dans les Etats membres une législation identique dans les matières choisies par le Conseil des Ministres.

L'instauration de ce régime juridique unique passe par l'édiction des normes supranationales appelées « actes uniformes ». Jusqu'au début de l'année 2021, à en croire KUMBU ki NGIMBI, l'OHADA a produit neuf actes uniformes touchant les sociétés commerciales et Groupement d'Intérêt Economique, les sûretés, le droit commercial général, les procédures simplifiées de recouvrement et des voies d'exécution, la médiation, la comptabilité et l'information financière, les sociétés coopératives, le transport de marchandises par route, l'arbitrage et les procédures collectives d'apurement du passif ${ }^{18}$.

Les instruments communautaires précités prévoient des règles de fond et de forme qui sont malheureusement méconnues par les textes législatifs et règlementaires édictés avant l'adhésion de la RDC à l'organisation pour l'harmonisation en Afrique du droit des Affaires. A titre illustratif, l'article 17 de la loi du 03/07/2001 cite les opérations boursières sur la liste des contentieux qui sont de la compétence matérielle du Tribunal de commerce alors qu'aujourd'hui l'Acte uniforme du 30/01/2014 reconnaît dans les Etats membres les bourses (marchés règlementés) et les marchés non réglementés.

17 L'article 36 de la loi du 03/07/2001 portant création, organisation et fonctionnement des Tribunaux de commerce cite les composants d'un acte d'opposition, entre autre, l'exposésommaire des moyens de l'opposant. En procédure pénale, cet élément n'est pas nécessaire. La simple déclaration suffit pour former opposition.

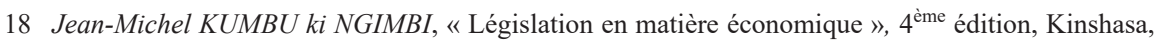
2020, pp.217-225. 
L'article 81 de l'Acte uniforme susvisé dispose : "sont réputés faire publiquement appel à l'épargne : les sociétés dont les valeurs mobilières sont admises à la négociation sur la bourse des valeurs d'un Etat partie, à dater de l'admission de ces titres; les sociétés ou toute personne qui offrent au public d'un Etat partie des valeurs mobilières dans les conditions énoncées à l'article 83 ci-après ${ }^{19}$. L'article 83 , quant à lui, renchérit : "l'offre au public de valeurs mobilières visés à l'article 81 ci-dessus est constituée par l'une des opérations : une communication adressée sous quelque forme et par quelque moyen que ce soit à des personnes et présentant une information sur les conditions de l'offre et sur les valeurs mobilières à offrir, de manière à mettre une personne en mesure d'envisager d'acheter ou de souscrire ces valeurs; un placement de valeurs mobilières par des intermédiaires financiers dans le cadre soit d'une émission, soit d'une cession $»^{20}$.

Le contenu de ces deux dispositions dénote la volonté du législateur communautaire de consacrer dans l'espace OHADA les marchés dématérialisés à côté des marchés boursiers comme c'est le cas en France et dansles autres Etat de l'Union Européenne depuis 2004. Pour des raisons de modernité, le législateur congolais devra élargir la compétence des Tribunaux de commerce aux opérations des marchés non réglementés. Une telle démarche favorisera une harmonisation des règles nationales aux normes supranationales qui, du reste, sont supérieures aux lois et règlements internes.

Toujours dans le même sens, la loi de 2001 est muette sur plusieurs questions de procédure qui émanent directement des actes uniformes. Les susdits actes uniformes, étant des règles communautaires, opèrent plusieurs renvoies au droit national. Le législateur ne traite pas cette matière pour le simple motif que la matière était méconnue en droit congolais lors de l'élaboration du texte législatif en vigueur. Il a été difficile pour le premier président de la Cour de cassation d'identifier la juridiction congolaise compétente dans les contestations d'exécution. L'arbitraire n'allait pas s'installer, même de façon sporadique, si la loi avait pris le soin de vider de manière définitive la compétence du juge de commerce dans ce domainematière comme c'est le cas en France.

En sus de la compétence, le statut des huissiers est aussi en antipode avec les nouvelles règles du droit congolais ${ }^{21}$. Comme nous l'avons dit dans les premières lignes de notre étude, les huissiers de justice œuvrent aujourd'hui comme des auxiliaires de justice indépendants de l'administration publique ${ }^{22}$. Une chambre provinciale a déjà vu le jour. A

19 Art. 81 de l'Acte uniforme sur les sociétés commerciales et groupement d'intérêt économique du 30 janvier 2014, JO/OHADA, Numéro du 30 janvier 2014.

20 Art. 83 de de l'Acte uniforme sur les sociétés commerciales et groupement d'intérêt économique du 30 janvier 2014, JO/OHADA,Numéro du 30 janvier 2014.

21 "Il est créé en République Démocratique du Congo, la profession d'huissier de justice dont l'organisation est régie par la présente loi »Art. 7 de la Loi n 16/011 du 15/07/2016 portant création et organisation de la profession d'huissier de justice, JORDC, Numéro spécial du 30/07/2016.

22 Art. 43 de la loi-organique $n^{\circ} 013 / 11-\mathrm{B}$ du 11/04/2013 portant organisation, fonctionnement et compétence des juridictions de l'ordre judiciaire, Kinshasa, JORDC, Numéro spécial du 04/05/2013. 
l'instar du Barreau et de l'ordre des experts comptables, les huissiers évoluent selon des règles déontologiques arrêtées par leurs autorités ordinales. La nouvelle législation pourra exclure les huissiers des fonctionnaires affectés au Tribunal de commerce conformément à la loi du 15/07/2016.

\section{CONCLUSION}

Somme toute, les insuffisances constatées dans la loi du 03/07/2001 portant création, organisation et fonctionnement des Tribunaux de commerce montrent la nécessité qu'il y a aujourd'hui de reformer profondément le texte législatif précité afin d'harmoniser son contenu au nouveau cadre juridique congolais, lequel cadre est fortement influencé d'abord par les normes supranationales nées des organisations communautaires dont la RDC est devenue membre après 2001, en suite par la politique en place depuis la promulgation de la Constitution de 2006.

En sus de l'organisation pour l'harmonisation en Afrique du Droit des Affaires qui via ses actes uniformes apportent des règles nouvelles, la communautépour le développement de l'Afrique australe impacte énormément la législation économique congolaise, principalement dans le secteur bancaire où la Banque Centrale du Congo a dû abandonner la loi $\mathrm{n}^{\circ} 005 / 2002 \mathrm{du}$ 07/05/2002 relative à la Constitution, à l'organisation et au fonctionnement de la Banque Centrale du Congo au profit d'une législation nouvelle, promulguée en 2018 (loi n $18 / 027$ du 13/12/2018) et contenant des dispositions presqu'identiques avec les textes juridiques qui régissent les Banques centrales des Etats membres de la SADEC.

$\mathrm{Au}$ niveau national, il sied de signaler que l'Etat congolais prend aussi ses propres initiatives, et ce, dans plusieurs secteurs de la vie économique. A titre illustratif, nous pouvons citer la loi-organique du 09/07/2018 relative à la liberté des prix et à la concurrence. L'article 84 du texte législatif précité dispose que seul le Tribunal de commerce est compétent pour juger les infractions prévues par la susdite loi-organique ${ }^{23}$. Les cas comme ceux-ci sont légions.

L'application dans le secteur économique de ces normes législatives issues soit des textes supranationaux, soit des initiatives nationales, peut préjudicier les prérogatives des justiciables en lieu et place d'améliorer le climat des affaires à travers la garantie d'une sécurité juridique et judiciaire, un des facteurs intrinsèques d'un Etat de droit. Si le susdit Etat de droit est celui dans lequel les gouvernants et les gouvernés sont soumis au bloc de textualité, il est très important pour les pouvoirs publics de fixer en avance les principes qui gouvernent d'abord l'organisation et le fonctionnement des juridictions, et ensuite, la procédure devant ces instances juridictionnelles.

23 «En cas de contentieux, les infractions aux dispositions de la présente loi sont de la compétence du tribunal de commerce. Le tribunal peut ordonner une expertise s'il juge que l'avis de l'Administration compétente est insuffisamment motivé " Art. 84 de la Loi-organique ${ }^{\circ} 18 / 020$ du 09 juillet 2018 relative à la Liberté des prix et à la concurrence, Kinshasa, JO/RDC, Numéro spécial, 23 juillet 2018, p.128. 
C'est ainsi que nous sommes d'avis que la loi du 03/07/2001 doit être réformée en vue de la converger avec le cadre juridique congolais actuel. Aussi, une réforme contribuera énormément à rendre précises les règles de compétence et de procédure devant les Tribunaux de commerce congolais ainsi que la problématique liée à l'organisation de cette juridiction.

\section{BIBLIOGRAPHIE}

\section{TEXTES OFFICIELS}

- Constitution de la République Démocratique du Congo du 18/02/2006 telle que modifiée par la Loi du 20/01/2011, Kinshasa, JO/RDC, Numéro spécial, 05/02/2011;

- Acte uniforme du 10/04/1998 portant organisation des procédures simplifiées de recouvrement des créances et des Voies d'Exécution, JO/OHADA, ํํㅇ, 01/06/1998;

- Acte uniforme sur les Sociétés commerciales et Groupement d'Intérêt économique du 30/01/2014, JO/OHADA, Numéro du 30/01/2014;

- Loi $\mathrm{n}^{\circ} 002-2001 \mathrm{du} 03 / 07 / 2001$ portant Création, organisation et fonctionnement des Tribunaux de commerce, Kinshasa, JORDC, ${ }^{\circ} 14,15 / 07 / 2001$;

- Loi-organique $\mathrm{n}^{\circ} 013 / 11-\mathrm{B}$ du 11/04/2013 portant organisation, fonctionnement et Compétence des Juridictions de l'ordre judiciaire, JORDC, Numéro spécial, 04/05/2013;

- Loi n¹6/011 du 15/07/2016 portant Création et Organisation de la Profession d'Huissier de Justice, Kinshasa, JO.RDC, Numéro spécial du 30/07/2016;

- Loi-organique $\mathrm{n}^{\circ} 18 / 020 \mathrm{du} 09 / 07 / 2018$ relative à la Liberté des prix et à la Concurrence, Kinshasa, JO/RDC, Numéro spécial, 23/07/2018;

- Arrêté d'Organisation Judiciaire n²99/79 du 20/08/1979 portant règlement intérieur des Cours, Tribunaux et Parquets;

\section{OUVRAGES}

- Emmanuel-Janvier LUZOLO BAMBI, Manuel de Procédure Pénale, Kinshasa, 2011;

- Gérard CORNU, Vocabulaire juridique, Paris, 2014;

- Jean-Michel KUMBU ki NGIMBI, Législation en matière économique, 4 èmédiion, Kinshasa, 2020;

- NYABIRUNGU mwene SONGA, Droit pénal général zaïrois, Kinshasa, 1989; 